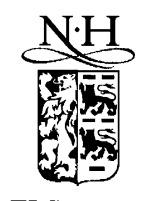

ELSEVIER
Journal of Monetary Economics 49 (2002) 435-453

\section{Journal of MONETARY EOONOMICS}

www.elsevier.com/locate/econbase

\title{
Government transaction policy and Gresham's law
}

\author{
Yiting Li* \\ Department of Economics, National Tsing Hua University, Hsin Chu 30013, Taiwan
}

Received 16 August 2000; received in revised form 19 February 2001; accepted 28 February 2001

\begin{abstract}
A search-theoretic model is used to study how legal restrictions may account for the fact that Gresham's law sometimes holds but other times fails. Legal restrictions is modeled here as policies that government adopts in the transactions with private agents, such as at what prices it accepts a currency in exchange for goods and at what ratios it conducts currency exchange. A government policy sufficiently favorable to light coins can induce the existence of an equilibrium where both coins circulate and light coins are even accepted at a premium. A policy favorable to heavy coins may increase their value to such an extent that they are hoarded from circulation, when this equilibrium did not exist without interventions. (C) 2002 Elsevier Science B.V. All rights reserved.
\end{abstract}

JEL classificaion: E40; E42; N10

Keywords: Commodity money; Gresham's law; Government policy

\section{Introduction}

The popular version of Gresham's law predicts that bad money drives out good money. ${ }^{1}$ Bad money is often referred to be under-weight coins or money overvalued

\footnotetext{
in I am very grateful to Randy Wright for many valuable suggestions and comments. I also thank an anonymous referee, participants in the Conference on Macroeconomics and International Finance at FuJen Catholic University and seminar participants at Chinese University of Hong Kong for comments. Any remaining errors are my own. Financial support from the National Science Council is acknowledged.

*Corresponding author. Tel.: + 886-3-5742722; fax: +886-3-5722476.

E-mail address: yli@mx.nthu.edu.tw (Y. Li).

${ }^{1}$ Some economists assert that a qualified version of Gresham's law requires a fixed exchange rate between the two monies (Friedman and Schwartz, 1963), but fixed exchange rate among various monies was not very often observed in history (see, for example, Rolnick and Weber, 1986; Velde et al., 1999).
} 
at the mint, and good money over-weight coins or money undervalued at the mint. Given what we know about history there had been times when bad money failed to drive out good, and many economists have questioned the empirical validity of Gresham's law. ${ }^{2}$

To study the applicability of Gresham's law, some models of commodity money, such as Sargent and Wallace (1983) and Sargent and Smith (1997), assume a fixed exchange rate between intrinsically distinct monies (called circulation by tale). However, in some of the medieval and early modern economies the powers of enforcement by the sovereign might not have been sufficient to enforce circulation by tale. Moreover, to explain circulation of various monies and determine endogenously their exchange value, we need a model which leaves circulation and exchange value of money as an equilibrium result rather than an assumption. To account for this, Velde et al. (1999) use a search-based model of commodity money without prior assumptions on exchange rates to study issues concerning the validity of Gresham's law and the mechanics of a debasement. They introduce private information by assuming that people are not always able to distinguish among various types of coins. In their model private information is the ingredient that allows for the simultaneous circulation of coins of different weights and at prices that do not necessarily reflect their metallic content.

The problem of recognizability of commodity money has prevailed at various times and in various places. However, we also observe that legal restrictions have played some role in affecting whether one object or another circulates as money and at what prices. ${ }^{3}$ Such policies include designating the legal tender status to a certain money and accepting only it in payment for custom duties or taxes. One of the historical examples in which legal restrictions played an important role in inducing a result contrary to the prediction of Gresham's law was the coinage of trade dollar and Bland dollar in U.S. during 1880. The light-weight Bland dollar was given the legal tender status. As a result, the Bland dollar and the heavier-weight trade dollar were current simultaneously, with the light-weight Bland dollar even circulated at a premium.

This paper uses a search-theoretic model to study how legal restrictions may account for the fact that Gresham's law sometimes holds but other times fails. I model legal restrictions as policies that government adopts in the transactions with private agents, such as at what prices it accepts a currency in exchange for goods and at what ratios it conducts currency exchange. The focus is to show how these policies affect which money (good or bad) circulates, the exchange rates among currencies and the deviation of exchange value of a currency from its intrinsic content.

\footnotetext{
${ }^{2}$ Rolnick and Weber (1986) describe several examples that seem to violate Gresham's law. For other observations and views that do not conform with Gresham's law, see references in Rolnick and Weber (1986).

${ }^{3}$ Adam Smith (1776) Smith, 1776 suggested that "A prince, who should enact that a certain proportion of his taxes should be paid in a paper money of a certain kind, might thereby give a certain value to this paper money; even though the term of its final charge and redemption should depend altogether upon the will of the prince".
} 
To be more specific, as in Aiyagari et al. (1996), Aiyagari and Wallace (1997) and $\mathrm{Li}$ and Wright (1998) I model government as a subset of agents, who are subject to the same random matching technology and other constraints as private agents, but who behave in an exogenous way regarding which money and at what price they accept in a trade, and at what ratios they conduct currency exchange. Currency exchange is not considered in Aiyagari and Wallace (1997) and Li and Wright (1998). ${ }^{4}$ Velde et al. (1999) simply ignore currency exchange for no good reasons, and I think this should be generalized. If we interpret currency exchange policy as quoting the ratio at the mint to make gold and silver bullion into coins or the conversion rate to redeem paper money, it would be interesting to know how these policies affect circulation and exchange value of monies. Moreover, in a general search monetary model with currencies of distinct prices, if we do not impose restrictions on exchange or matching technology, currency exchange always takes place in equilibrium. ${ }^{5}$ Hence, from the viewpoint of exploring more implications of search monetary models I like to consider a policy on currency exchange in this paper. $^{6}$

Note that this paper is different from some previous studies on commodity money that simply assume a fixed exchange rate (imposed by legal restrictions) between monies with distinct metallic content. The model considered here allows us to determine which money circulates and at what price, and to study how big the sovereign powers it needs to achieve an equilibrium where monies circulate at the exchange rate that does not necessarily reflect difference in their intrinsic content. Thus, legal restrictions in the present model is not an assumption to impose a fixed exchange rate, but rather, a factor to be formulated so that we can investigate its effects on the circulation and exchange value of monies.

In this paper commodity money derives value beyond intrinsic content from its role as a medium of exchange that mitigates the double-coincidence-of-wants problem. I construct a variety of examples to display the equilibrium outcomes that conform or violate Gresham's law. First, it is shown that monies with distinct metallic content may circulate side by side without government interventions. I find an equilibrium in which light coins are even valued higher in exchange than heavy coins. This occurs when the difference in their intrinsic content is small. People's belief in the exchange value of a currency can induce deviation of its market price from intrinsic content to such an extent that light coins manage to circulate at a

\footnotetext{
${ }^{4}$ Currency exchange is considered in two-country-two-currency search-theoretic models such as Matsuyama et al. (1993) and Zhou (1997). In Matsuyama et al. (1993) currency exchange takes place only in a mixed equilibrium when money holders are indifferent from holding domestic and foreign currency. Zhou (1997) considers preference shocks to induce currency exchange in equilibrium.

${ }^{5}$ For example, Velde et al. (1999) assume that buyers never trade with other buyers. Aiyagari and Wallace (1997) and Li and Wright (1998) assume that money holders never meet with each other.

${ }^{6}$ Some other related search-theoretic papers of dual assets include Curtis and Waller (2000) and Wallace (2000). Curtis and Waller (2000) study how government punishment and enforcement policies affect the circulations and prices of legal and illegal fiat currencies. Wallace (2000) explains how the differences in transaction velocity of assets are affected by the size of their exogenous real dividends. The basic model presented here shares many features with Wallace (2000) if we interpret the intrinsic value of a currency as the exogenous real dividend of an asset.
} 
higher price than heavy coins. However, the deviation would not go too far, as it still respects the commodity nature of monies. As the difference gets bigger and the metallic content of heavy coins is below some threshold, the only equilibrium involves simultaneous circulation of both coins with heavy coins circulating at a premium. If the metallic content of heavy coins is above some threshold, they will be hoarded from circulation and Gresham's law is working. In this case, heavy coins are too intrinsically valuable to be used as a medium of exchange.

I then proceed to consider government policies that conduct currency exchange at a pre-specified ratio or accept both heavy and light coins at the same par value. One example that I find is an exception to Gresham's law, due to government interventions. A policy favorable to light coins can induce the existence of an equilibrium where both coins circulate and light coins are even accepted at a premium. Government policy can affect private agent's incentives to such an extent that the market exchange ratio contradicts what implied by the intrinsic content of coins. It is also shown that a policy favorable to heavy coins may increase their value to such an extent that they are hoarded from circulation, when this equilibrium did not exist without interventions. Thus, I attribute the working of Gresham's law in this case to legal restrictions. I then consider a policy that government accepts heavy and light coins at the same par value to offer an alternative explanation for how policy affects people's incentives to bring coins to the mint in return for lighter coins after a debasement.

The rest of this paper proceeds as follows. Section 2 presents the basic model. Section 3 discusses the existence and properties of various equilibria. Section 4 introduces government and discusses the effects of government transaction policies on circulation and exchange value of monies. Section 5 concludes with a summary of the main results and a discussion of some historical examples.

\section{The basic model}

Time is discrete and the horizon is infinite. There is a $[0,1]$ continuum of infinitely lived agents. Specialization, which motivates gains from trade but also makes trade difficult, is modeled here as follows. There are $N$ distinct, perfectly divisible but nonstorable goods and $N$ types of agents with equal population. Each type is specialized in consumption and production in the following way: a type $i$ agent consumes good $i$ and produces good $i+1$ (modulo $N$ ), for $i=1,2, \ldots, N$, where $N \geqslant 3$. Thus, there is no double coincidence of wants.

When agent $i$ consumes $q$ units of his consumption good he enjoys utility $u(q)$; when he produces $q$ units of his production good he suffers disutility $c(q)$. I normalize $c(q)=q$ with no loss of generality. Production is instantaneous. The utility function $u(q)$ is defined on $[0, \infty)$, is strictly increasing and twice differentiable, and satisfies $u(0)=0, u^{\prime}(0)=\infty$, and $u^{\prime \prime}(q)<0$. Also, there is a $\hat{q}>0$ such that $u(\hat{q})=\hat{q}$. Each agent is endowed with production technology according to his type. Each type $i$ agent maximizes expected discounted utility with a discount rate $r$. 
There are two kinds of storable objects that can potentially serve as media of exchange, called money 1 and money 2, with stock $M_{j}$ of money $j$ and $M=$ $M_{1}+M_{2}<1$. At the initial date, a fraction $M_{j} \in(0,1)$ of each type of agents are endowed with money $j$. There is an instantaneous utility $v_{j}$ from holding money $j$, and the source of the value comes from possession of the metal or selling the specie in the world market. ${ }^{7}$ Since this paper studies issues around commodity money, I interpret $v_{j}$ as the "intrinsic value" or "metallic content" of money $j$. Suppose $v_{2}>$ $v_{1}>0$, and so money 1 is referred as light coins and money 2 heavy coins. Or, if the par value of both coins is "a dollar" then money 1 is referred as bad money and money 2 good money.

Agents meet pairwise according to a random-matching process with arrival rate $\beta$. Each agent's trading history is private information to the agent. Private credit is ruled out and all trades are quid pro quo. It is assumed that money holders always trade the whole unit of their money. Each agent can hold at most one unit of one money at a time.

Note that types are identical in the sense that $r, u(q)$ and $c(q)$, the measure of each type, the matching process, and storage capacity for money are identical for all types. It is also assumed that the initial distribution of money holdings is symmetric across types. These specifications make it sensible to consider equilibria that are symmetric across types, and those are the kind of equilibria that I consider in this paper.

\subsection{Bargaining and exchange}

Given the above specifications, at each date each agent holds either one unit of one money or nothing. Agents in pairwise meetings bargain. If the outcome of bargaining involves exchange, production and consumption occurs. I use a simple version of the bilateral bargaining approach used in Shi (1995) and Trejos and Wright (1995): assume that the consumer makes a take-it-or-leave-it offer to the producer. A consequence of this bargaining rule is that consumers extract all of the gains from trade. The take-it-or-leave-it offer is a special case of the generalized Nash bargaining solution, and it simplifies the analysis considerably.

In each pairwise meeting trade involving production occurs only when a type $i$ agent meets a type $i+1$ agent. We call this a single-coincidence meeting. Given the upper bound of unity on money holdings, there are two potential trading situations in single-coincidence meetings: when the consumer has a unit of money and the producer does not, and when the consumer has a more valuable money (in terms of exchange value, not intrinsic value) than the producer has. In the former situation,

\footnotetext{
${ }^{7}$ The flow value of possessing money could be interpreted as a reduced form for a story such as the following one. Money holders buy goods from the world market, in which coins are accepted by weight. There is a probability of meeting foreign traders with whom money holders can trade and derive utility from consuming the goods. The more complicated modeling on the intrinsic value of money will not change the main results of this paper. To make the analysis more focus on the issue of interest, I did not model foreign trade as mentioned in the story.
} 
money may be traded for some amount of goods. In the latter situation, the more valuable money may be traded for the less valuable money plus some amount of production as side payment. To prevent ambiguity, hereafter I refer producers as agents who have no money in hand.

When a money $j$ holder meets a producer who produces his consumption good, the amount $q_{j}$ may be produced in exchange for money. When a money $j$ holder meets a money $k$ holder who produces his consumption good, the amount $q_{j k}$ may be produced and monies change hands (i.e., there occurs currency exchange). I define here that a money circulates when it is accepted in exchange solely for goods as well as in currency exchange; i.e., when it is universally acceptable. ${ }^{8}$

\section{Equilibria}

Let $V_{j}$ be the lifetime expected utility to an agent holding money $j$. The implications of the bargaining rule that producers do not gain in a trade give us the following results: The discounted utility from beginning a period with no money is zero, and the terms of trade satisfy

$$
\begin{aligned}
& q_{j}=V_{j}, \\
& q_{j k}=V_{j}-V_{k} .
\end{aligned}
$$

Since consumer's take-it-or-leave-it offers make the agents who produce indifferent between accepting and rejecting the offers, whether a money circulates thus depends on buyers' willingness to spend when they meet producers.

Let $\omega_{j}$ be the probability that a money $j$ holder is willing to spend his money in a trade with a producer. The best response condition is described as

$$
\omega_{j}= \begin{cases}1 & \text { if } u\left(q_{j}\right)-V_{j} \geqslant 0, \\ 0 & \text { if } u\left(q_{j}\right)-V_{j}<0 .\end{cases}
$$

Hence, (1) and (3) imply that a money $j$ holder is willing to trade his money for goods if $0 \leqslant q_{j} \leqslant \hat{q}$. Let $\sigma_{j}$ denote the probability that money $j$ holder is willing to trade his money for money $k$ plus some amount of production, $q_{j k}$. The best response condition is

$$
\sigma_{j}= \begin{cases}1 & \text { if } u\left(q_{j k}\right)+V_{k}-V_{j} \geqslant 0 \\ 0 & \text { if } u\left(q_{j k}\right)+V_{k}-V_{j}<0\end{cases}
$$

Conditions (2) and (4) imply that currency exchange occurs if $0 \leqslant q_{j k} \leqslant \hat{q}$. ${ }^{9}$

\footnotetext{
${ }^{8}$ This definition implies that if a money is acceptable only in currency exchange, it is not qualified as a circulating medium of exchange. This definition is suitable for the purpose of this paper, since in some historical episodes when Gresham's law applied good money was still used in few occasions.

${ }^{9}$ In general money holders may use mixed strategies when they are indifferent; i.e., $u\left(q_{j}\right)=q_{j}$ and $u\left(q_{j k}\right)=q_{j k}$, which implies $q_{j}=\hat{q}$ and $q_{j k}=\hat{q}$. This does not provide additional insight and, hence, we consider only the case of pure strategies.
} 
In what follows we let $\beta / N=\alpha$, which measures how severe the transaction friction is. The value functions thus satisfy

$$
\begin{aligned}
& r V_{1}=v_{1}+\alpha(1-M) \max _{\omega_{1}} \omega_{1}\left[u\left(q_{1}\right)-V_{1}\right]+\alpha M_{2} \max _{\sigma_{1}} \sigma_{1}\left[u\left(q_{12}\right)+V_{2}-V_{1}\right], \\
& r V_{2}=v_{2}+\alpha(1-M) \max _{\omega_{2}} \omega_{2}\left[u\left(q_{2}\right)-V_{2}\right]+\alpha M_{1} \max _{\sigma_{2}} \sigma_{2}\left[u\left(q_{21}\right)+V_{1}-V_{2}\right] .
\end{aligned}
$$

Notice that the first maximization problem in (5) and (6) implies that the buyer chooses whether or not to spend his money when he meets a producer, and the second maximization problem implies that the agent with more valuable money chooses whether or not to engage in currency exchange.

Definition 1. A symmetric steady state equilibrium is a vector of value functions $V=\left(V_{1}, V_{2}\right)$, quantities $q=\left(q_{1}, q_{2}, q_{12}, q_{21}\right)$ and strategies $(\omega, \sigma)=\left(\omega_{1}, \omega_{2}, \sigma_{1}, \sigma_{2}\right)$ such that (i) $V$ satisfy (5) and (6); (ii) $q$ satisfy (1) and (2); and (iii) $(\omega, \sigma)$ satisfy (3) and (4).

Equilibria are different in whether heavy and light coins are used in exchange solely for goods, and whether there occurs currency exchange. The following lemma establishes the result that under a general environment such as the one considered here (e.g., no restrictions on exchange and matching technology), there is always currency exchange if there are multiple circulating monies.

Lemma 1. When both types of monies circulate, there is always currency exchange.

Proof. See the appendix.

I characterize equilibria in terms of the vector of strategies $\left(\omega_{1}, \omega_{2}, \sigma_{1}, \sigma_{2}\right)$. Note that $\omega_{j}$ and $\sigma_{j}$ can take value of 0 or 1 so potentially there are 16 equilibria. Some cases can be ruled out and we are left with only seven types of equilibria. ${ }^{10}$ By Lemma 1 the case $\left(\omega_{1}, \omega_{2}, \sigma_{1}, \sigma_{2}\right)=(1,1,0,0)$ is ruled out. Note that when currency exchange takes place, it is either heavy coins in exchange for light coins plus some amount of goods or vise versa, but never both. Thus I rule out all cases with $\sigma=(1,1)$. Also note that when heavy coins are too dear to be spent solely for goods and only light coins circulate, if there is currency exchange, it must be the holders of light coins to produce side payment to exchange for heavy coins (see the appendix for proof). Hence, the case $(1,0,1,0)$ is ruled out. Furthermore, fundamentals do not support equilibria in which heavy coins circulate but light coins are hoarded (see the appendix for proof). Thus, I rule out all the cases with $\omega=(0,1)$. In the following subsections I discuss the existence and implications of equilibria.

\footnotetext{
${ }^{10}$ The seven equilibria consist of $\left(\omega_{1}, \omega_{2}, \sigma_{1}, \sigma_{2}\right)=(1,1,0,1),(1,1,1,0),(1,0,0,1),(1,0,0,0)$, $(0,0,0,1),(0,0,1,0),(0,0,0,0)$.
} 


\subsection{Equilibria with two circulating currencies}

\subsubsection{By-weight equilibrium $\left(\omega_{1}, \omega_{2}, \sigma_{1}, \sigma_{2}\right)=(1,1,0,1)$}

In this equilibrium both heavy and light coins circulate and heavy coins are accepted at a higher price. This is called by-weight equilibrium because the exchange value of both coins reflects their intrinsic content. I show in the appendix that if $v_{2}<r \hat{q}-\alpha M_{1}\left[u\left(\hat{q}-q_{1}^{*}\right)-\left(\hat{q}-q_{1}^{*}\right)\right]$, both monies circulate and heavy coins are traded for light coins plus the amount of $\operatorname{good} q_{21}^{*} \in(0, \hat{q})$, where $q_{21}^{*}=q_{2}^{*}-q_{1}^{*}$, and $q_{j}^{*}$ denotes the exchange value of money $j$. The existence condition implies that if the utility of possessing heavy coins plus the expected flow return from currency exchange is less than $r \hat{q}$, heavy coins will circulate and command a premium. That is, when the metallic content of heavy coins is not too big, people will enjoy the gains of using it as a medium of exchange rather than hoard it from circulation. This equilibrium is an exception to Gresham's law.

\subsubsection{Anti-by-weight equilibrium $\left(\omega_{1}, \omega_{2}, \sigma_{1}, \sigma_{2}\right)=(1,1,1,0)$}

Now consider an equilibrium in which both currencies circulate simultaneously but heavy coins are accepted at a discount. It is shown in the appendix that if $v_{1}<r \hat{q}-\alpha M_{2}\left[u\left(\hat{q}-q_{2}^{*}\right)-\left(\hat{q}-q_{2}^{*}\right)\right], v_{2}<r \hat{q}$ and $v_{2}-v_{1}$ is small enough, both monies circulate and light coins are traded for heavy coins plus the amount of good $q_{12}^{*} \in(0, \hat{q})$, where $q_{12}^{*}=q_{1}^{*}-q_{2}^{*}$. Hence, I conclude that as long as the difference in the intrinsic value of both currencies is small enough, there exists an equilibrium in which light coins are valued higher than the heavy ones in the market. Note that this result is contrary to what is found in Velde et al. (1999) that heavy coins always circulate at a higher price than light coins.

To illustrate some empirical implications of this equilibrium, let's assume for this moment $v_{1}=0$ and call money 1 "fiat money" and money 2 some sort of commodity money, say, copper coins (so the difference in their intrinsic value is small). The existence of this equilibrium implies that people may value intrinsically worthless paper higher than copper coins in exchange simply because they believe so. The belief constitutes a "fiat component" of the monetary value of a currency. How far can the exchange value deviate from intrinsic value? The present model predicts that the "fiat component" of a currency can support deviation of the market exchange ratio from that implied by intrinsic content as long as the difference in intrinsic value of competing currencies is not too big, and how big depends on the amount of monies in circulation, discount rate and other parameters.

Note that the by-weight equilibrium and anti-by-weight equilibrium coexist for some parameter values. This multiplicity of equilibria is a common feature in searchbased models of money, and it is a self-fulfilling phenomenon.

\subsection{Equilibria with one circulating currency}

In this subsection I show that when the intrinsic value of a money makes it too dear to be spent solely for goods, it will be hoarded from circulation. The market fails to generate a big enough premium on good money for it to circulate as a 
medium of exchange. ${ }^{11}$ This type of equilibria represent a situation which conforms to Gresham's law.

\subsubsection{Single-currency equilibrium with currency exchange $\left(\omega_{1}, \omega_{2}, \sigma_{1}, \sigma_{2}\right)=(1,0,0,1)$}

In this equilibrium, holders of money 2 pass the trade with producers and engage only in two steps of exchange - trade heavy coins for light coins plus some amount of output and then use the light coins to buy goods later. This equilibrium exists when $v_{1}<r \hat{q}<v_{2}<r\left(\hat{q}+q_{1}^{*}\right)$, where $q_{1}^{*}$ is the market price of money 1 . That is, if the intrinsic content of money 2 is too big to be used solely for goods but not too big to generate an acceptable term of trade in currency exchange, there exists an equilibrium in which money 1 is the unique circulating medium of exchange and money 2 is used only in currency exchange.

\subsubsection{Single-currency equilibrium with no currency exchange $\left(\omega_{1}, \omega_{2}, \sigma_{1}, \sigma_{2}\right)=$ $(1,0,0,0)$}

This equilibrium exists when $v_{1}<r \hat{q}$ and $v_{2}-v_{1}>r \hat{q}+\alpha(1-M)\left[u\left(q_{1}^{*}\right)-q_{1}^{*}\right]$. If the intrinsic value of money 2 is too big to generate acceptable terms of trade in exchange for money 1, people will hoard it from circulation and from currency exchange. Heavy coins, due to the superior intrinsic content, are "too valuable" to be used as a medium of exchange. People rather use it as a commodity or enjoy the value of possessing the metal. ${ }^{12}$

\subsection{Equilibria with no circulating currency}

In this type of equilibria both monies are too dear to derive acceptable terms of trade in exchange solely for goods, i.e., $v_{1}>r \hat{q}, v_{2}>r \hat{q}$. There may or may not be currency exchange, depending on the difference of intrinsic content of coins. If the difference in metallic content is not too big, $v_{2}-v_{1}<r \hat{q}$, there exists an equilibrium in which heavy coins command a premium in currency exchange. If $v_{2}-v_{1}$ is small enough there exists an equilibrium where heavy coins trade for light coins at a discount. If the metallic content of both coins and their difference are all too big to generate acceptable terms of trade, there exists a degenerate outcome that no trade ever occurs.

\section{Government policy}

It has been shown that the circulation and exchange value of a currency are determined not only by its intrinsic content but also by people's belief. The belief

\footnotetext{
${ }^{11}$ Rolnick and Weber (1986) argue that bad money drives good money out of circulation only when the costs of using good money at a premium are significant. They describe a situation in which small denomination good money is hoarded due to the rounding problem.

${ }^{12} \mathrm{We}$ can imagine that there are goldsmith or exporters in this economy who buy heavy coins and melt them down for industrial use or ship them aboard and pay the holder $v_{2} / r$ in return.
} 
adds to a currency a "fiat component" that enables deviation of the monetary value from intrinsic content. In this section I discuss how government policy affects the fiat component and monetary value of a currency.

I model government agents in a way similar to Aiyagari and Wallace (1997) and Li and Wright (1998). In Li and Wright (1998) we demonstrate the effects of government transaction policy on the determination of which objects are accepted as media of exchange and at what prices. In this paper I consider a new policy instrument - exchange rate policy, which specifies at what ratio government trades one currency for another. ${ }^{13}$ I assume that a fraction of the population $\gamma$ constitutes a special class called government agents. They are in all respects like private agents except that they adopt exogenous trading rules rather than strategies based on maximizing behavior. ${ }^{14}$ The objective is to see how government policy affects the trading strategies that private agents choose endogenously, and hence the exchange value of currencies and the set of equilibria.

\subsection{Exchange rate policy}

The exchange rate policy specifies at what ratio government trades one currency for another. This policy can be interpreted as specifying the ratio of minting bullion into coins in a commodity money system, or the rate of converting paper money into specie in a convertible money system. Assume that this policy specifies an exogenously fixed quantity of goods $q_{j k}^{\mathrm{g}}$ which government agents produce to exchange one unit of money $k$ for money $j$. The quantity $q_{j k}^{\mathrm{g}}$ can be interpreted as the official exchange rate of money $j$ to money $k$. Although I do not explicitly model the objective of government policy, this policy can be motivated as an instrument to establish a currency favored by government as the medium of exchange. For example, government that wishes to establish convertible paper money as the medium of exchange may manipulate the conversion rate to affect the market price of paper money.

\subsubsection{Exchange rate policy favorable to money 1}

Suppose that without government interventions the unique equilibrium is a byweight equilibrium where both monies circulate and money 1 is accepted at a discount. Now consider a policy that government agents accept money 1 at a premium of $q_{12}^{\mathrm{g}}$ (and behave as private agents in other respects). Note that this policy adds to money 1 some value which derives from the opportunity of trading with government that accepts the inferior money at a premium.

Since the exchange patterns specified in this economy are symmetric across private and government agents, the resulting distributions of money holdings for private and

\footnotetext{
${ }^{13}$ Alternatively I could have assumed that, as in Aiyagari et al. (1996), government sometimes rejects to trade one kind of money for another. This policy can be motivated by a situation such as government makes it easier to redeem paper money for specie (e.g., establishes more posts for redemption).

${ }^{14}$ One should interpret $\gamma$ as capturing the importance of government in the economy, or the frequency with which private agents interact with the public sector, rather than measuring number of individuals who "work for the government".
} 
government agents are identical. The flow return to money 2 holders is given by (6), and the flow value to money 1 holders satisfies

$$
\begin{aligned}
r V_{1}= & v_{1}+\alpha(1-\gamma)\left\{(1-M) \max _{\omega_{1}} \omega_{1}\left[u\left(q_{1}\right)-V_{1}\right]\right. \\
& \left.+M_{2} \max _{\sigma_{1}} \sigma_{1}\left[u\left(q_{12}\right)+V_{2}-V_{1}\right]\right\} \\
& +\alpha \gamma\left\{(1-M) \max _{\omega_{1}} \omega_{1}\left[u\left(q_{1}\right)-V_{1}\right]+M_{2}\left[u\left(q_{12}^{\mathrm{g}}\right)+V_{2}-V_{1}\right]\right\} .
\end{aligned}
$$

Note that private agents will reject trade with government if $q_{12}^{\mathrm{g}}$ is too small. Hence, I consider only $q_{12}^{\mathrm{g}}$ policy such that $u\left(q_{12}^{\mathrm{g}}\right)+V_{2}-V_{1}>0$ in equilibrium. The main results regarding $q_{12}^{\mathrm{g}}$ policy are summarized as follows.

Proposition 1. The exchange rate policy that government accepts money 1 at a premium of $q_{12}^{\mathrm{g}}$ affects the existence of equilibrium and exchange rate. If $q_{12}^{\mathrm{g}}$ is big enough (or, equivalently, given $q_{12}^{\mathrm{g}}$ the size of government $\gamma$ is big enough) there exists an equilibrium where money 1 circulates at a premium, which did not exist without government interventions. Even if the policy does not affect the type of equilibrium, by using $q_{12}^{\mathrm{g}}$ the government can reduce the discount on money 1 .

Proof. See the appendix.

This result implies that when the official exchange rate is significantly favorable to money 1 or, equivalently, when the sovereign powers is sufficient enough, government policy can affect private agent's incentives to such an extent that the market exchange ratio contradicts what implied by the intrinsic content of coins. Even $q_{12}^{\mathrm{g}}$ or $\gamma$ is not big enough to change the type of equilibrium, it can still reduce the discount on light coins in private transactions.

Some historical examples described in Rolnick and Weber (1986) are consistent with our basic predictions. During 1870s in U.S. Bland dollar and trade dollar circulated side by side and lighter-weight Bland dollar was accepted at a premium. The Bland dollar was given legal tender status. Contrary to the prediction of Gresham's law, the lighter weight Bland dollar not only failed to drive out the heavier-weight trade dollar but also managed to circulate at a higher price. Greenbacks were legal tender notes issued by Congress in U.S. to finance the Civil War. During the early stage of greenback era (1862-79) in the West, greenbacks did not drive out specie but rather, were current at a discount. Good money and bad money circulated side by side with the good one current at a higher price. $^{15}$ The present analysis offers an explanation for these historical examples, if we interpret policy $q_{12}^{\mathrm{g}}$ as government's favorable treatment for money with legal tender status.

\footnotetext{
${ }^{15}$ Greenfield and Rockoff (1995) dispute whether this example counts against Gresham's law by noting that Californians may consider greenbacks foreign exchange not money.
} 


\subsubsection{Exchange rate policy favorable to money 2}

Suppose that without government interventions the unique equilibrium is a byweight equilibrium. Now consider a policy that government trades money 1 plus the quantity $q_{21}^{\mathrm{g}}$ in exchange for money 2 . This policy changes the flow value to the holders of money 2 to

$$
\begin{aligned}
r V_{2}= & v_{2}+\alpha(1-\gamma)\left\{(1-M) \max _{\omega_{2}} \omega_{2}\left[u\left(q_{2}\right)-V_{2}\right]\right. \\
& \left.+M_{1} \max _{\sigma_{2}} \sigma_{2}\left[u\left(q_{21}\right)+V_{1}-V_{2}\right]\right\} \\
& +\alpha \gamma\left\{(1-M) \max _{\omega_{2}} \omega_{2}\left[u\left(q_{2}\right)-V_{2}\right]+M_{1}\left[u\left(q_{21}^{\mathrm{g}}\right)+V_{1}-V_{2}\right]\right\}
\end{aligned}
$$

and the value to the holders of money 1 is given by (5). Obviously, this policy works to affect the premium on heavy coins. Let $q_{21}^{o}$ denote the equilibrium exchange ratio without policy. One can show that if $q_{21}^{\mathrm{g}}>(<) q_{21}^{o}$, then the market exchange ratio is increased (decreased) by this policy. That is, if government treats money 2 more (less) favorable than the market does, it will increase (decrease) the premium on money 2 in private transactions.

One interesting question is that, would this policy add too big a fiat component to money 2 to make it too dear to trade solely for goods? If this is the case then money 2 would be used only in currency exchange, and the flow value to holders of money 2 becomes

$$
r V_{2}=v_{2}+\alpha M_{1}\left\{(1-\gamma) \max _{\sigma_{2}} \sigma_{2}\left[u\left(q_{21}\right)+V_{1}-V_{2}\right]+\gamma\left[u\left(q_{21}^{\mathrm{g}}\right)+V_{1}-V_{2}\right]\right\} .
$$

I find that when $q_{21}^{\mathrm{g}}$ is big enough, there exists an equilibrium where heavy coins are hoarded from circulation. Note that this equilibrium did not exist without government interventions. This result thus implies that Gresham's law phenomenon may be due to government's favorable treatment to a currency which makes it too dear to be put in circulation. ${ }^{16}$

The main results are summarized in the following proposition.

Proposition 2. The exchange rate policy that government accepts money 2 at a premium of $q_{21}^{\mathrm{g}}$ affects the existence of equilibrium and exchange rate. If $q_{21}^{\mathrm{g}}$ is sufficiently big, the only equilibrium is the one where money 2 is hoarded from circulation, which did not exist without government interventions. Even if the policy

\footnotetext{
${ }^{16}$ It is impossible for this policy to drive money 2 totally out of the market, since the fiat component must be added through trading with government agents. One may conjecture a situation where money 2 holders pass the trade with any private agents and engage only in currency exchange with government. However, this may result in government agents filled up with money 2 and so they are no longer able to take money 2 from private agents. One way to avoid this situation is that government agents take in money 2 and destroy it or remint it into money 1. Another policy to avoid this is that government trades money 2 for money 1 with private agents (even though private agents would not do the same) and through which government releases money 2 back to the market.
} 
does not affect the type of equilibrium, by using $q_{21}^{\mathrm{g}}$ the government can affect the premium on money 2.

\subsection{Debasement and "A dollar is a dollar" policy}

Starting from the medieval age governments had debased coins and acquired a large amount of seigniorage revenues. Historically, a debasement was an offer by the mint to swap light coins for heavy coins. Usually, the mint would give more light coins than the number of heavy coins turned in, but the total metallic content of light coins was less than that of heavy coins, and the difference was seigniorage. Obviously, an operation of debasement lowered the metallic content of coins that people brought in, then why would they voluntarily bring in old coins for reminting after a debasement? Velde et al. (1999) consider private information as an element to provide an incentive for some agents to bring coins to the mint despite the loss in intrinsic content. Here I provide an example to show how government transaction policy affects private agents' incentives to do so.

Consider a case where government, following a debasement, accepts both old and new coins at the same par value. This captures the phenomenon that coins were accepted by tale domestically but accepted aboard by weight. As in Velde et al. (1999) I model an operation of debasement here by assuming that the mint trades one light coin plus some amount of output as side payment for one heavy coin. It is assumed that the output which serves as side payment can be consumed immediately and generates an instantaneous utility of $\eta$.

Suppose that we were in an economy where there were $M$ heavy coins circulating as the medium of exchange and no light coins. Now government conducts a debasement and announces that it accepts all types of coins at the par value of $q_{g}$. After debasement the amount of light and heavy coins depends on how many agents with heavy coins go to the mint, which in turn depends on the side payment and the price at which government accepts old and new coins. Note that under the present setup everyone has either one coin or zero coins, whether they go to the mint or not.

There are three types of equilibria characterized by the amount of light coins: (i) $M_{1}=0$ (no reminting); (ii) $M_{1}=M$ (complete reminting); (iii) $M_{1} \in(0, M)$ (partial reminting). First note that since $\eta$ is the side payment for turning in heavy coins to the mint in exchange for light coins, an equilibrium with $M_{1}>0$ must have the feature that heavy coins are valued higher than light coins in private transactions. That is, it must be a by-weight equilibrium. Second, for agents being indifferent from whether or not to bring old coins to the mint, the side payment must satisfy $V_{1}+\eta=$ $V_{2}$. Since the existence of currency exchange in private transactions implies $q_{j k}=$ $V_{j}-V_{k}, \eta$ must be equal $q_{21}$ in the equilibrium with $M_{1} \in(0, M)$. Also note that contrary to Velde et al. (1999) where the equilibrium with $M_{1} \in(0, M)$ must be a bytale equilibrium, ${ }^{17}$ in the present model the equilibrium with $M_{1}>0$ must be a byweight equilibrium.

\footnotetext{
${ }^{17}$ They define the by-tale equilibrium as one in which both coins are accepted at the same price at least in some trades.
} 


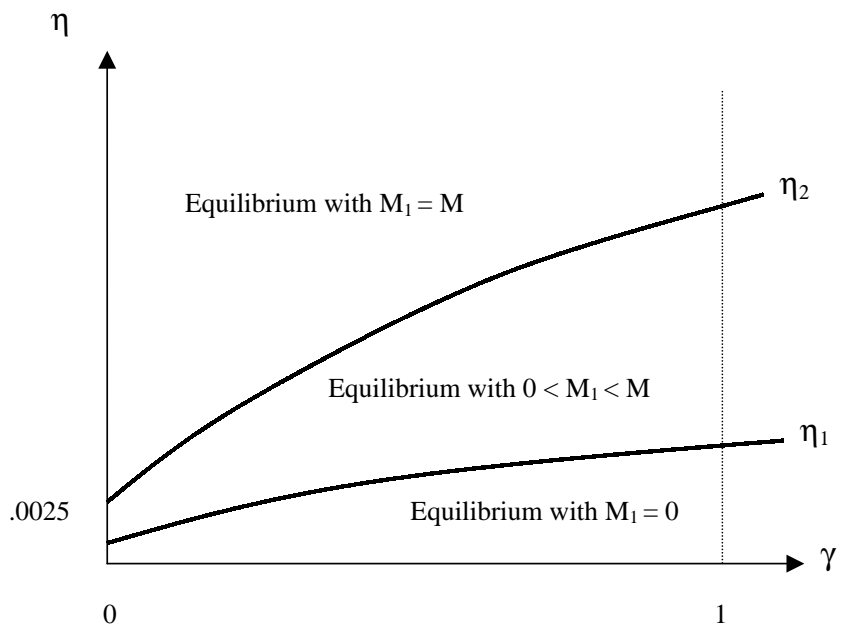

Fig. 1. Existence of equilibria after debasement. Equilibrium with $M_{1}=M$ exists if $\eta \geqslant \eta_{2}$. Equilibrium with $M_{1}=0$ exists if $\eta \leqslant \eta_{1}$. Equilibrium with $0<M_{1}<M$ exists if $\eta_{1}<\eta<\eta_{2}$. Example: $u(q)=q^{1 / 2}$. Parameters: $r=0.1, \alpha=1, v_{1}=0.04, v_{2}=0.05, M=0.2, q_{\mathrm{g}}=0.98$.

I show existence of equilibria in the appendix and summarize the main results in the following proposition.

Proposition 3. There are three types of equilibria, which exist in the regions shown in Fig. 1. The equilibrium with $M_{1}=0$ exists if $\eta \leqslant \eta_{1}$, equilibrium with $M_{1}=M$ exists if $\eta \geqslant \eta_{2}$, and equilibrium with $M_{1} \in(0, M)$ exists if $\eta_{1}<\eta<\eta_{2}$, where the critical values of the side payment, $\eta_{1}$ and $\eta_{2}$, are functions of the size of government $\gamma$.

The example $u(q)=\sqrt{q}$ is used to illustrate $\eta_{1}$ and $\eta_{2}$ as functions of $\gamma$, and the existence regions of equilibria in Fig. 1. From Fig. 1 we know that given $\gamma$ there is a nonempty region of side payment $\eta$ such that at least some agents bring coins to the mint. I also do some comparative statics for the equilibrium with $M_{1} \in(0, M)$. As we increase $q_{g}$ (or $\eta$ ), the exchange value of both coins and $M_{1}$ are increased. This implies that as government increases the par price to accept both coins (or the side payment to debase coins), it will increase their exchange value in private transactions and also enhance private agents' incentive to bring coins to the mint and, therefore, increase the amount of light coins after debasement. ${ }^{18}$

\footnotetext{
${ }^{18}$ Since there is currency exchange in the private market, as long as government provides just enough side payment, agents would be willing to bring coins to the mint even without government policy of accepting both coins at the same par value. Nevertheless, our analysis still provides some predictions as how government's treatment of distinct monies affects the monetary value of coins and people's incentives to bring coins to the mint after debasement.
} 


\section{Conclusions}

This paper has studied the effects of policies that specify at what prices government accepts monies and at what ratio it conducts currency exchange. It is established that government's treatment of distinct monies may be a factor to account for the fact that Gresham's law sometimes holds but other times fails. If government policy is too much favorable to light coins, it is possible to entail an outcome in which light coins circulate at a higher price than heavy coins. Also, a policy sufficiently favorable to heavy coins may add too big a fiat component to the coins and make it too dear to be put in circulation. If we interpret this policy as one adopted by a foreign country that prefers a particular metal, say, silver, then this paper predicts that silver coins will disappear from circulation because they will be exported or sold as bullion to the foreign country.

I have also illustrated some equilibria where there are few or even no circulating media of exchange, which resemble the situation of a currency shortage - coins are too dear to be spent in the market. Trade is severely reduced because commodity money is hoarded from monetary use. Under this situation it would be valuable to adopt a policy to generate more and lighter-weight coins to facilitate trade. ${ }^{19}$ To pursue this issue, one may need a model in which government adopts a recoinage policy that generates more coins, and there is a constant inflow of agents that absorb the increase of money supply so that everyone has either one coin or zero coins. One can use such model to discuss how recoinage policy affects the frequency of trade, the price level, and the role of government in providing sufficient intermediation to facilitate trade.

Greenfield and Rockoff (1995) describe a historical example in which government's manipulation of the official exchange rate (the mint ratio) affected the market ratio and circulation of coins. In the early 19th century of U.S., gold was undervalued at the mint given the prevailing market prices (so it was good money). Gold coins were not in circulation. Even though there was continued minting of gold, much of the gold coinage was exported and much of the rest was sold as bullion. After the Congress reduced the weight of the gold dollar in 1834, thereby raising the mint ratio, gold coins were made overvalued in the mint. Because the difference between the mint ratio and the market ratio was small, gold coins did not drive silver coins out of circulation. However, as more gold discoveries in the late 1840s and early 1850s increased the market value of silver dollars from its intrinsic content, gold became dominant circulating medium. The predictions of this paper are consistent with the observations in Greenfield and Rockoff (1995): if government exchange rate policy does not result in too big a difference in the mint ratio and market ratio, both coins circulate. As the policy makes a money too valuable to be used as a medium of exchange (such as silver dollar of the late 1840s in the example), it will be hoarded from circulation.

\footnotetext{
${ }^{19}$ Wallace and Zhou (1997) describe a situation of currency shortage due to indivisibility of money.
} 


\section{Appendix}

Proof of Lemma 1. We prove Lemma 1 by contradiction. Suppose both monies circulate and there is no currency exchange. Hence, the value functions become

$$
\begin{aligned}
& r q_{1}=\alpha(1-M)\left[u\left(q_{1}\right)-q_{1}\right]+v_{1}, \\
& r q_{2}=\alpha(1-M)\left[u\left(q_{2}\right)-q_{2}\right]+v_{2} .
\end{aligned}
$$

Given $v_{2}>v_{1}>0$ and $v_{1}, v_{2}<r \hat{q}$ one can solve for $q_{1}^{*} \in[0, \hat{q}], q_{2}^{*} \in[0, \hat{q}]$, and $q_{1}^{*}<q_{2}^{*}$. Now suppose a money 2 holder deviates to propose $\tilde{q}_{21}$ such that $\tilde{q}_{21}=q_{2}^{*}-q_{1}^{*}+\varepsilon$ to a money 1 holder, $\varepsilon \rightarrow 0$. Since accepting this offer makes money 1 holder better off, it will not be rejected. The deviation also makes money 2 holder better off since $u\left(\tilde{q}_{21}+q_{1}^{*}-q_{2}^{*}\right)>0$. Thus, there occurs currency exchange, a contradiction.

$(\omega, \sigma)=(1,0,1,0)$ is not an equilibrium: By contradiction, suppose $(\omega, \sigma)=$ $(1,0,1,0)$. The value functions are

$$
\begin{aligned}
& r V_{1}=\alpha(1-M)\left[u\left(q_{1}\right)-V_{1}\right]+\alpha M_{2}\left[u\left(q_{12}\right)+V_{2}-V_{1}\right]+v_{1}, \\
& r V_{2}=v_{2}
\end{aligned}
$$

and $V_{1}=q_{1}$. Since money 2 is hoarded, $V_{2}>\hat{q}$, which is satisfied if $v_{2}>r \hat{q}$. To induce money 2 holder to produce and surrender his money, money 1 holder proposes $q_{12}$ such that $V_{1}-V_{2}-q_{12} \geqslant 0$. Note that for money 1 to circulate, $q_{1}^{*} \in[0, \hat{q}]$. Since $q_{1}^{*}<\hat{q}<V_{2}$, it is impossible to find $q_{12}^{*} \in[0, \hat{q}]$ for the trade to occur.

$\omega=(0,1)$ does not constitute an equilibrium: By contradiction, suppose $(\omega, \sigma)=$ $(0,1,0,0)$. Thus, $V_{1}>\hat{q}$, which is satisfied if $v_{1}>r \hat{q}$. Since money 2 circulates,

$$
r q_{2}=\alpha(1-M)\left[u\left(q_{2}\right)-q_{2}\right]+v_{2}
$$

and $q_{2} \in[0, \hat{q}]$, which imply $v_{2}<r \hat{q}$, a contradiction to the assumption $v_{1}<v_{2}$. By a similar argument, $(\omega, \sigma)=(0,1,0,1)$ is not an equilibrium.

Now we check that $(\omega, \sigma)=(0,1,1,0)$ is not an equilibrium. In this case,

$$
\begin{aligned}
& r V_{1}=\alpha M_{2}\left[u\left(q_{12}\right)+q_{2}-V_{1}\right]+v_{1}, \\
& r V_{2}=\alpha(1-M)\left[u\left(q_{2}\right)-V_{2}\right]+v_{2},
\end{aligned}
$$

where $q_{12}=V_{2}-V_{1}$. Note that $\omega=(0,1)$ implies $V_{1}>\hat{q}$ and $q_{2}<\hat{q}$. Hence, there exists no value for $q_{12} \in[0, \hat{q}]$ such that $\sigma_{1}=1$.

The existence condition for equilibrium $\left(\omega_{1}, \omega_{2}, \sigma_{1}, \sigma_{2}\right)=(1,1,0,1)$ : We substitute the candidate strategic parameters into the value functions (5) and (6), and then verify that those strategies are best responses. Given $\left(\omega_{1}, \omega_{2}, \sigma_{1}, \sigma_{2}\right)=(1,1,0,1)$, we have the following value functions:

$$
\begin{aligned}
& r q_{1}=\alpha(1-M)\left[u\left(q_{1}\right)-q_{1}\right]+v_{1}, \\
& r q_{2}=\alpha(1-M)\left[u\left(q_{2}\right)-q_{2}\right]+\alpha M_{1}\left[u\left(q_{21}\right)+q_{1}-q_{2}\right]+v_{2} .
\end{aligned}
$$


From (A.1) one can solve for $q_{1}=q_{1}^{*} \in[0, \hat{q}]$ if $v_{1} \leqslant r \hat{q}$. Let $x=q_{2}-q_{1}^{*}$. We rewrite (A.2) in terms of $x$ as $F(x)=H(x)$ where $F(x)=r\left(x+q_{1}^{*}\right)$ and $H(x)=\alpha(1-$ $M)\left[u\left(x+q_{1}^{*}\right)-\left(x+q_{1}^{*}\right)\right]+\alpha M_{1}[u(x)-x]+v_{2}$. Since $H(0)=F(0)+\left(v_{2}-v_{1}\right)$ and $H^{\prime}(0) \rightarrow \infty$, it follows that $F(0)<H(0)$ and $F(x)<H(x)$ for $x$ close to 0 . Also, $H(\hat{q})<F(\hat{q})$ if $v_{2} \leqslant r \hat{q}$. Since $H$ and $F$ are continuous, there exists at least one $x \in(0, \hat{q})$ such that $F(x)=H(x)$. Let $q_{21}^{*}$ denote this $x$. Since $q_{21}^{*} \in(0, \hat{q})$, the trade of money 2 for money 1 plus the amount of production $q_{21}^{*}$ is optimizing.

Now we need to verify that given others' strategies $\omega_{2}=1$ is a best response. Given $q_{1}^{*}$ we can rewrite (7) as $q_{2}=D\left(q_{2}\right)$ where $D\left(q_{2}\right)=\left\{\alpha(1-M) u\left(q_{2}\right)+\right.$ $\left.\alpha M_{1}\left[u\left(q_{21}^{*}\right)+q_{1}^{*}\right]+v_{2}\right\} /\left[r+\alpha\left(1-M+M_{1}\right)\right]$. Thus, if $D(\hat{q})<\hat{q}$, i.e., $\quad v_{2}<r \hat{q}-$ $\alpha M_{1}\left[u\left(\hat{q}-q_{1}^{*}\right)-\left(\hat{q}-q_{1}^{*}\right)\right]$, there exists a quantity $q_{2}^{*} \in[0, \hat{q}]$ such that money 2 in exchange solely for goods.

The existence condition for equilibrium $\left(\omega_{1}, \omega_{2}, \sigma_{1}, \sigma_{2}\right)=(1,1,1,0)$ : Given the candidate strategic parameters the value functions are

$$
\begin{aligned}
& r q_{1}=\alpha(1-M)\left[u\left(q_{1}\right)-q_{1}\right]+\alpha M_{2}\left[u\left(q_{12}\right)+q_{2}-q_{1}\right]+v_{1}, \\
& r q_{2}=\alpha(1-M)\left(u\left(q_{2}\right)-q_{2}\right)+v_{2} .
\end{aligned}
$$

Again, $q_{2}^{*} \in[0, \hat{q}]$ if $v_{2} \leqslant r \hat{q}$. Let $x=q_{1}-q_{2}^{*}$. We rewrite (A.3) as $F(x)=H(x)$ where $F(x)=r\left(x+q_{2}^{*}\right)$ and $H(x)=\alpha(1-M)\left[u\left(x+q_{2}^{*}\right)-\left(x+q_{2}^{*}\right)\right]+\alpha M_{2}[u(x)-x]+v_{1}$. Note that $H(0)<F(0)$ and $H(\hat{q})<F(\hat{q})$. Since $H^{\prime}(0) \rightarrow \infty$, if $H(0)$ is close enough to $F(0)$, which requires $v_{2}-v_{1}$ is small enough (how small depends on the curvature of utility function and other parameters), it is possible to find a $x \in(0, \hat{q})$ such that $F(x)=H(x)$.

By similar argument as in previous proof, $\omega_{1}=1$ is a best response if $v_{1}<r \hat{q}-\alpha M_{2}\left[u\left(\hat{q}-q_{2}^{*}\right)-\left(\hat{q}-q_{2}^{*}\right)\right]$.

Proof of Proposition 1. Rewrite the flow value to money 1 holder as

$$
\begin{aligned}
r q_{1}= & \alpha(1-M)\left[u\left(q_{1}\right)-q_{1}\right]+\alpha M_{2}\left[u\left(q_{12}\right)+q_{2}-q_{1}\right] \\
& +\alpha \gamma M_{2}\left[u\left(q_{12}^{\mathrm{g}}\right)-u\left(q_{12}\right)\right]+v_{1} .
\end{aligned}
$$

Using the same reasoning in the proof for equilibrium $(1,1,1,0)$ and $(1,1,0,1)$, we rewrite the above equation as $F(x)=H(x)$ where $F(x)=r\left(x+q_{2}^{*}\right)$ and $H(x)=$ $\alpha(1-M)\left[u\left(x+q_{2}^{*}\right)-\left(x+q_{2}^{*}\right)\right]+\alpha M_{2}[u(x)-x]+\alpha \gamma M_{2}\left[u\left(q_{12}^{\mathrm{g}}\right)-u(x)\right]+v_{1}$. Denote $\tilde{v}_{1}(x)=\alpha \gamma M_{2}\left[u\left(q_{12}^{\mathrm{g}}\right)-u(x)\right]+v_{1}$, which could be interpreted as money 1's new intrinsic value. Note that $F(0)=H(0)+v_{2}-\tilde{v}_{1}(0)$. If $q_{12}^{\mathrm{g}}$ is big enough, then $v_{2}-$ $\tilde{v}_{1}(0)<0$ even though $v_{2}>v_{1}$, and so $H(0)>F(0)$. Also, $H(\hat{q})<F(\hat{q})$ because $v_{2} \leqslant r \hat{q}$. Since $H$ and $F$ are continuous, there exists at least one $x \in(0, \hat{q})$ such that $F(x)=$ $H(x)$. Let $q_{12}^{*}$ denote this $x$. Since $q_{12}^{*} \in(0, \hat{q})$, the trade of money 1 for money 2 plus the amount of production $q_{12}^{*}$ is optimizing.

To show that when $q_{12}^{\mathrm{g}}$ is not big money 1 still circulates at a discount but the discount is reduced by policy, to simplify follow the proof in equilibrium $(1,1,0,1)$ and note that now $H(0)=F(0)+v_{2}-\tilde{v}_{1}(0)$. If $q_{12}^{\mathrm{g}}$ is not big, then $v_{2}-\tilde{v}_{1}(0)>0$, and so $H(0)>F(0)$. Hence, there exists at least one $x \in(0, \hat{q})$ such that $F(x)=H(x)$. Let $q_{21}^{*}$ denote this $x$. Since $q_{21}^{*} \in(0, \hat{q})$, the trade of money 2 for money 1 plus the amount 
of production $q_{21}^{*}$ is optimizing. Note that policy $q_{12}^{\mathrm{g}}$ moves down the curve of $H(x)$ and so the discount on money $1, q_{21}^{*}$, is reduced.

Proof of Proposition 3. (i) $M_{1}=0$ : If no one goes to the mint then it must be that government does not provide enough incentives, i.e., $V_{1}+\eta \leqslant V_{2}$. The value of holding money 2 is $V_{2}$, and if an agent deviates and goes to the mint, he gets the side payment $\eta$ plus the value of holding money $1, V_{1}$, where

$$
\begin{aligned}
& r V_{2}=v_{2}+\alpha(1-M)\left\{(1-\gamma)\left[u\left(q_{2}\right)-V_{2}\right]+\gamma\left[u\left(q_{g}\right)-V_{2}\right]\right\}, \\
& r V_{1}=v_{1}+\alpha(1-M)\left\{(1-\gamma)\left[u\left(q_{1}\right)-V_{1}\right]+\gamma\left[u\left(q_{g}\right)-V_{1}\right]\right\} .
\end{aligned}
$$

Substituting (A.4) and (A.5) into the condition $V_{1}+\eta \leqslant V_{2}$ one can solve for the critical value of $\eta_{1}$ as a function of parameters such that when $\eta \leqslant \eta_{1}$ there exists an equilibrium where no one brings coins to the mint.

(ii) $M_{1}=M$ : If everyone brings money 2 to the mint, then $V_{1}+\eta \geqslant V_{2}$. Agents who do so receive the side payment and the value of holding money 1 , as defined in (A.5). If an agent deviates and hold the heavy coins, he gets the flow return

$$
\begin{aligned}
r V_{2}= & v_{2}+\alpha(1-M)\left\{(1-\gamma) \max \left[u\left(q_{2}\right)-V_{2}, 0\right]+\gamma\left[u\left(q_{g}\right)-V_{2}\right]\right\} \\
& +\alpha M_{1} \max \left[u\left(q_{21}\right)+V_{1}-V_{2}\right] .
\end{aligned}
$$

Substituting (A.5) and (A.6) into the condition $V_{1}+\eta \geqslant V_{2}$ one can solve for the critical value of $\eta_{2}$ as a function of parameters such that when $\eta \geqslant \eta_{2}$ there exists an equilibrium where everyone goes to the mint.

(iii) $M_{1} \in(0, M)$ : The flow value of holding money 1 and money 2 satisfies (A.5) and (A.6), respectively. One can substitute (A.5) and (A.6) into $V_{1}+\eta=V_{2}$ and solve for the conditions on $\eta$ such that $M_{1} \in(0, M)$. From numerical examples we find that if $\eta_{1}<\eta<\eta_{2}$ there exists an equilibrium with $M_{1} \in(0, M)$.

\section{References}

Aiyagari, S.R., Wallace, N., 1997. Government transaction policy, the medium of exchange and welfare. Journal of Economic Theory 74, 1-18.

Aiyagari, S.R., Wallace, N., Wright, R., 1996. Coexistence of money and interest-bearing securities. Journal of Monetary Economics 37, 397-419.

Curtis, E.S., Waller, C.J., 2000. A search-theoretic model of legal and illegal currency. Journal of Monetary Economics 45, 155-184.

Friedman, M., Schwartz, A.J., 1963. A Monetary History of the United States, 1867-1960. Princeton University Press, Princeton, NJ.

Greenfield, R.L., Rockoff, H., 1995. Gresham's law in nineteenth-century America. Journal of Money, Credit and Banking 27, 1086-1098.

Matsuyama, K., Kiyotaki, N., Matsui, A., 1993. Towards a theory of international currency. Review of Economic Studies 60, 283-307.

Li, Y., Wright, R., 1998. Government transaction policy, the media of exchange and prices. Journal of Economic Theory 81, 290-313.

Rolnick, A.J., Weber, W.E., 1986. Gresham's law or Gresham's fallacy? Journal of Political Economy 94, 185-199. 
Sargent, T.J., Wallace, N., 1983. A model of commodity money. Journal of Monetary Economics 12, 163-182.

Sargent, T.J., Smith, B.D., 1997. Coinage, debasements, and Gresham's laws. Economic Theory 10, $197-226$.

Shi, S., 1995. Money and prices: a model of search and bargaining. Journal of Economic Theory 67 , 467-498.

Smith, A., 1776. The Wealth of Nations. Richard D. Irwin, Inc., Homewood, IL.

Trejos, A., Wright, R., 1995. Search, bargaining, money and prices. Journal of Political Economy 103, $118-141$.

Velde, F.R., Weber, W.E., Wright, R., 1999. A model of commodity money, with application to Gresham's law and the debasement puzzle. Review of Economic Dynamics 2, 291-323.

Wallace, N., 2000. A model of the liquidity structure based on asset indivisibility. Journal of Monetary Economics 45, 55-68.

Wallace, N., Zhou, R., 1997. A model of a currency shortage. Journal of Monetary Economics 40, 555-572.

Zhou, R., 1997. Currency exchange in a random search model. Review of Economic Studies 64, 289-310. 